\title{
Editorials
}

\section{Sleep apnoea:}

\author{
no laughing matter
}

Obstructive sleep apnoea (OSA) is often considered amusing, probably because of its association with snoring and obesity. Although the related 'Pickwickian' syndrome was identified in the early 20th century, OSA remained invisible until the development of nocturnal electroencephalographic (EEG) and respiratory monitoring in the mid 1960s, which allowed physicians to identify individual respiratory events. OSA was initially thought to be rare, but epidemiological studies showed it to be a common problem, affecting around $4 \%$ of the population' of whom $80 \%$ are not diagnosed.

As early studies included only men, OSA was thought to be a uniquely male disease, but more recent population studies have shown that the male:female ratio is about 2-3:1. As the ratio in sleep clinic populations is around 8:1, women are not being identified and referred. ${ }^{2}$ An important risk factor for OSA is obesity, with over $30 \%$ of patients with morbid obesity suffering from severe OSA. Rising obesity rates are likely to increase prevalence. However, OSA is also highly prevalent in patients with diabetes (35\%), hypertension (40\%), resistant hypertension (70\%), and atrial fibrillation (45\%). 3.4 Family history is important, and patients often report close family members suffering from OSA.

\section{WHAT IS OSA?}

OSA is caused by intermittent obstruction of the upper airways during sleep. Airway obstruction is multifactorial, and anatomical problems (such as tonsillar hypertrophy). fatty deposits in the pharynx, and weakness of dilatory muscles in the pharynx all play a role. As the airways narrow, airflow becomes turbulent, leading to snoring. As the airways narrow more, respiratory volume decreases, and respiratory effort increases leading to hypopnoeas. Once the airways close, airflow stops which leads to apnoea. Both apnoeas (total obstructions) and hypopnoeas (partial obstructions) are considered significant because they have the potential to fragment sleep, cause falls in oxygen saturation, and lead to activation of the sympathetic nervous system.

OSA has been confirmed as an important, independent risk factor for cardiovascular disease, and it is now certain that untreated severe OSA increases mortality and cardiovascular morbidity, ${ }^{5}$ and seriously impairs vigilance, performance, and quality of life. ${ }^{6}$ The gravity of OSA is measured by the number of respiratory events per hour of sleep using the apnoea-hypopnoea index (AHI): an $\mathrm{AHI}<5$ is considered normal, and $>30$ severe OSA.

\section{OSA SYMPTOMS}

If asked to describe a typical patient with OSA, GPs may think of an obese middleaged man with snoring, respiratory pauses, and daytime sleepiness; however, the clinical picture is more complex. The patient is not necessarily male or middle aged: OSA can occur in children, usually in association with tonsillar hypertrophy. Only 60\% are overweight and nocturnal symptoms, such as snoring, respiratory pauses, restless sleep, and nocturia, are not always reported - a patient living alone may not know that they snore. Daytime symptoms such as sleepiness, morning headache, poor performance at school or work, and mood changes are often attributed to other causes. The classic triad of snoring, daytime sleepiness, and respiratory pauses is common and found in $7.5 \%$ of the French adult population.?

Clinical examination in OSA is often unhelpful, although a high body mass index, an increased neck circumference $1>42 \mathrm{~cm}$ men and $>39 \mathrm{~cm}$ women), and ear-nosethroat abnormalities (such as retrognathia, macroglossia, and tonsillar hypertrophy) are useful pointers. Sleepiness is often measured using the Epworth Sleepiness Scale, where a score of $>10$ indicates sleepiness and $>15$ indicates severe sleepiness. Severe sleepiness is a contraindication to driving.

\section{SHOULD WE SCREEN FOR OSA?}

The high prevalence and effectiveness of management of sleep apnoea are potent arguments for screening, but current screening tools are neither sensitive nor specific, $^{8}$ and so GPs' clinical acumen remains crucial in detecting and referring suspected cases of OSA. OSA is a recently recognised pathology, and has been on the medical syllabus in most countries from the 1990s, so it is not surprising that studies in France, ${ }^{9}$ US ${ }^{10}$ and Canada ${ }^{11}$ have shown that GPs' knowledge about sleep apnoea and their ability to detect OSA in simulated patients is poor. ${ }^{12}$ There is evidence from studies over the last decade in France that knowledge is improving, linked to attendance at continuing medical education sessions.

\section{MANAGEMENT OF SLEEP APNOEA}

There is considerable variation in sleep service provision both within and between countries. Gaps in provision are frequent, and acess to services often slow. Diagnosis of sleep apnoea requires nocturnal sleep studies, either at home or in hospital. Several techniques are used and there is considerable international variation. The gold standard investigation is hospital polysomnography which combines EEG and respiratory monitoring. Depending on the equipment used, ambulatory nocturnal respiratory monitoring has been shown to have adequate sensitivity and specificity, and is much less expensive. As a result, ambulatory techniques are accepted in many countries as an alternative to polysomnography. In resource-limited settings oximetry alone is an alternative, although sensitivity is limited, as only severe cases of OSA with frequent desaturations will be detected. Sleep apnoea is treatable, and correction of OSA leads to rapid normalisation of daytime sleepiness, improvement of vigilance, performance, and fatigue.

While weight loss improves OSA, with the exception of bariatric surgery, significant weight loss is rarely achieved in practice. Bypassing the upper airways obstruction via a tracheostomy was the only method

\section{"The patient is not necessarily male or middle aged: OSA can occur in children, usually in association with tonsillar hypertrophy.}


available until the development of continuous positive airway pressure (CPAP) by Sullivan in 1981. CPAP has since been shown to normalise cardiovascular mortality and morbidity in severe apnoeics both in secondary ${ }^{13}$ and primary care ${ }^{14}$ and is the current first line of treatment. Indications for treatment depend on the $\mathrm{AHI}$ and vary internationally.

While CPAP is effective, it is not easy to use. Problems with the interface between the machine and the patient are frequent, masks often leak, and if overtightened may damage skin. Patients often need a lot of support in the first few weeks, and treatment can be optimised by providing a sympathetic ear, motivating and advising patients to keep using their machine, to try the machine during the day to habituate, and to go back to their OSA team to sort out mask difficulties or problems falling asleep. Varying dropout rates internationally (55-80\% still treated after 2 years) may reflect the availability of patient support.

More recently, alternative modes of treatment have been developed. Mandibular advancement devices increase the diameter of the upper airway by advancing the lower jaw and have been shown to be successful in reducing sleep apnoea. The success rate of surgery depends on the initial indications: in the case of tonsillar hypertrophy in children, tonsillectomy is highly effective, but in other cases using different techniques results are variable. Over a lifetime of sleep apnoea, patients may try several forms of treatment, and knowing that alternatives are available (even if potentially less effective) is important, especially for younger patients who may find CPAP incompatible with their lifestyle.

Is OSA over diagnosed? Probably not: current evidence indicates under rather than over diagnosis. Is OSA overtreated in certain countries? Possibly: we know that the percentage of patients treated per head of population treated varies between countries. This reflects not only differences in OSA prevalence (related to obesity) and in service provision, but also variations in national treatment recommendations and health insurance rules (surgery may be covered but not CPAP or mandibular advancement devices). High CPAP treatment rates in certain countries may be linked to better service provision and lower treatment thresholds, but may also be due to lack of alternative treatment modalities, and poor audit of services with a failure to detect patients who have dropped out of treatment. Overall, it is likely that OSA is undertreated in most countries.

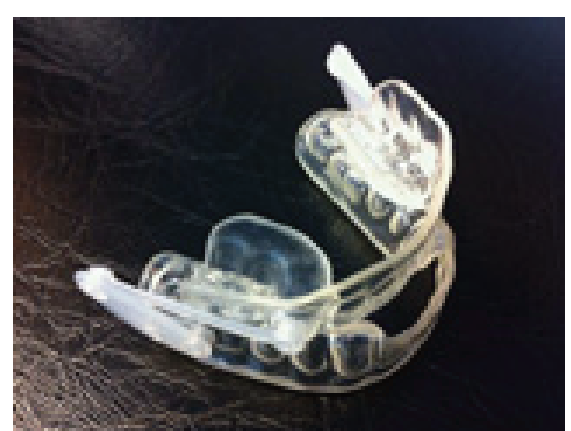

Mandibular advancement devices are increasingly used in the the management of sleep apnoea.

\section{CONCLUSION}

OSA is highly prevalent, eminently treatable, and many patients are not diagnosed. GPs should be aware of the high prevalence in certain patient groups, recognise that presentation may be atypical, and actively ask patients about symptoms associated with sleep apnoea. Patients should be referred to their local sleep service, reserving urgent referrals for patients who have a high risk for OSA, are sleepy, and in whom reduced vigilance poses a risk to themselves or others (for example, heavy goods drivers). Identifying and developing good links with local OSA services is essential.

\section{Sarah Hartley,}

Hôpital Poincaré, Service d'Explorations

Fonctionelles, Garches, France.

\section{Josselin Vachon,}

Faculté de Médecine Paris Descartes, Department of General Practice, Paris, France.

\section{Olivier Benainous,}

Faculté de Médecine Paris Descartes, Department of General Practice, Paris, France.

\section{Provenance}

Commissioned; not externally peer reviewed.

DOI: 10.3399/bjgp11X583001

\section{ADDRESS FOR CORRESPONDENCE}

\section{Sarah Hartley}

Hôpital Poincaré, Service d'Explorations

Fonctionelles, 104 Boulevard Raymond Poincaré, Garches, 78170, France.

E-mail: sarah.hartleydwanadoo.fr

\section{REFERENCES}

1. Young T, Palta M, Dempsey J, et al. The occurrence of sleep-disordered breathing among middle-aged adults. N Engl J Med 1993; 328: 1230-1235.

2. Ye L, Pien GW, Ratcliffe SJ, Weaver TE. Gender differences in obstructive sleep apnea and treatment response to continuous positive airway pressure. J Clin Sleep Med 2009: $\mathbf{5}$ 512-518.

3. Nieto FJ, Young TB, Lind BK, et al. Association of sleep-disordered breathing, sleep apnea, and hypertension in a large community-based study. Sleep Heart Health Study. JAMA 2000; 283: $1829-1836$

4. Goncalves SC, Martinez D, Gus M, et al. Obstructive sleep apnea and resistant hypertension: a case-control study. Chest 2007 132: 1858-1862.

5. Young T, Finn L, Peppard PE, et al. Sleep disordered breathing and mortality: eighteenyear follow-up of the Wisconsin sleep cohort. Sleep 2008; 31: 1071-1078.

6. Beebe DW, Groesz L, Wells C, et al. The neuropsychological effects of obstructive sleep apnea: a meta-analysis of norm-referenced and case-controlled data. Sleep 2003; 26: 298-307.

7. Meslier N, Vol S, Balkau B et al. [Prevalence of symptoms of sleep apnea syndrome. Study of a French middle-aged population]. Rev Mal Respir 2007; 24: 305-313

8. Abrishami A, Khajehdehi A, Chung F. A systematic review of screening questionnaires for obstructive sleep apnea. Can J Anaesth 2010; 57: 423-438.

9. Pontier S, Matiuzzo M, Mouchague JM, et al. [General practice management of obstructive sleep apnea in the Midi-Pyrenees]. Rev Mal Respir 2007; 24: 289-297.

10. Rosen RC, Zozula R, Jahn EG, Carson JL. Low rates of recognition of sleep disorders in primary care: comparison of a communitybased versus clinical academic setting. Sleep Med 2001; 2: 47-55.

11. Chung SA, Jairam S, Hussain MR, Shapiro CM Knowledge of sleep apnea in a sample grouping of primary care physicians. Sleep Breath 2001; 5: 115-121.

12. Reuveni $H$, Tarasiuk A, Wainstock $T$, et al. Awareness level of obstructive sleep apnea syndrome during routine unstructured interviews of a standardized patient by primary care physicians. Sleep 2004; 27: 1518-1525.

13. Giles TL, Lasserson TJ, Smith BH, et al. Continuous positive airways pressure for obstructive sleep apnoea in adults. Cochrane Database Syst Rev 2006; 3: CD001106.

14. Di Guardo A, Profeta G, Crisafulli C, et al. Obstructive sleep apnoea in patients with obesity and hypertension. Br J Gen Pract 2010; 60(574): 325-328. 